\title{
Parental Alcoholism and Coping: A Comparison of Female Children of Alcoholics With Female Children of Nonalcoholics
}

\author{
Virginia A. Kelly and Jane E. Myers
}

\begin{abstract}
The authors assessed potential differences between children of alcoholics and children of nonalcoholics in depression and choice of coping strategies. Although significant group differences were detected for level of depression, mean depression scores for both groups fell within the no depression range on the Beck Depression Inventory. No significant differences in coping strategies were detected.
\end{abstract}

T he National Institute on Alcohol Abuse and Alcoholism (NIAAA) has estimated that $28,000,000$ Americans have at least one alcoholic parent (NIAAA, 1985). One in every six individuals is being or has been raised by at least one alcoholic parent. Clinically based literature related to the potential effects of parental alcoholism emerged with some regularity throughout the $1980 \mathrm{~s}$ (Beattie, 1987; Black, 1981). The bulk of this literature focused on a characterization of children of alcoholics (COAs), which was based on the accumulation of clinical information. This particular literature base described the population of COAs as potentially at risk for a set of fairly predictable social and psychological problems that may exist well into adult life.

An empirically based body of literature pertaining to COAs has since evolved. Evidence suggests that a well-defined, empirically based characterization of COAs remains elusive. The frequent incidence of confounding variables beyond researchers' control has led to equivocal findings throughout the literature; however, in a thorough review of the literature on COAs, Russell, Henderson, and Blume (1985) concluded that this population seems to be a high-risk group.

Specifically, the literature suggests that COAs exhibit lower levels of cognitive functioning (Plescia-Pikus, Long-Sutter, \& Wilson, 1988), lower levels of overall psychological well-being (Svanum \& McAdoo, 1991), decreased levels of self-esteem (Berkowitz \& Perkins, 1988), poor sociability (Calder \& Kostiniuk, 1989), and an external locus-of-control orientation (Prewett, Spence, \& Chaknis, 1981). In addition, the research to date has unequivocally linked parental alcoholism with higher levels of depression when COAs were compared with a variety of matched cohort groups (Calder \& Kostiniuk, 1989: Tweed \& Ryff, 1991).

This same set of social and psychological characteristics has also been shown to affect various aspects of the processes associated with stress and coping (Lazarus \& Folkman, 1984). More specifically, the literature on stress and coping suggests that locus-of-control orientation, level of overall psychological well-being, and level of depression are related to choice of coping strategies (Folkman \& Lazarus, 1985; Kolenc, Hartley, \& Murdock, 1990; Pearlin \& Schooler, 1978; Thompson, 1981).

Lazarus and Launier (1978) suggested that the ability to successfully cope with stress is paramount to an overall sense of psychological well-being. Although COAs are at risk for low well-being, the investigation of potentially unique coping strategies among this group represents an important topic of study. A review of relevant studies described below yielded a lack of empirically based information on this topic, prompting the design of the current study to explore potential differences in coping strategies between COAs and children of nonalcoholics (CONAs).

The literature on stress and coping among COAs is scarce. Martin (1991) found that COAs reported using a higher number of evasive, fatalistic, palliative, and self-reliant coping strategies than CONAs. Scavnicky-Mylant (1990) reported that young adult COAs demonstrated a developmental delay in the use of certain coping strategies when compared with CONAs. In addition, COAs were significantly more likely to use two unique coping strategies: reversed emotive coping, defined as focusing on the emotions of another as opposed to focusing on one's own emotions, and reversed confrontative coping, defined as the act of comforting another as opposed to seeking comfort for oneself.

The evidence presented led us to hypothesize that COAs will cope with stress differently than CONAs. Our study was designed to investigate specific differences between the two groups in choice of coping strategies. The sample for this study was restricted to females to eliminate the confounding influence of gender (see Berkowitz \& Perkins, 1988; Borgatta, 1965). In addition, because higher levels of depression have been unequivocally linked to both parental alcoholism and choice of coping strategies, the potential difference in level of depression between COAs and CONAs was assessed. As a result, depression was controlled for within the applied statistical model, where scores on the Beck Depression Inventory (BDI; Beck, Ward, Mendelson, Mock, \& Erbaugh, 1961) were entered as a covariate. Thus, the primary research questions addressed were these:

1. Do female COAs show higher levels of depression than female CONAs as evidenced by the BDI?

2. Do female COAs differ from female CONAs in coping with family-related stress, as evidenced by the Ways of Coping Checklist-Revised (WCCL-R) (Folkman \& Lazarus, 1985)?

3. Can coping strategy profiles be used to predict COA and CONA group membership?

\section{METHOD}

\section{Procedure}

Lazarus and Folkman (1984) and Folkman and Lazarus (1985) defined coping as the dynamic and interdependent relationship between the person, the environment, and the stressful encounter. Given the central role of interaction in coping, these authors suggested that the 
construct of coping be studied within the context of specific stressful encounters. For the COA, the most profound impact of parental alcoholism occurs within the context of family interactions. Therefore, this study addressed differences in choice of strategies used to cope with stressful family situations.

Using the procedure developed and endorsed by Lazarus and Folkman (1984), potential participants were asked to describe briefly a stressful encounter that had taken place within their family in the 6-month period before data collection. They were then asked to respond to the WCCL-R based on their described encounter. This set of responses is referred to as "actual stressful encounters." To assist in controlling for detected differences attributable to differences in actual stressful encounters, as opposed to differences in choice of coping strategies, participants were also asked to complete the WCCL- $R$ in response to a prepared vignette. The vignette required them to imagine that they were the child in a scenario in which the parents of a traditional-aged college student harshly reprimand their child for staying out until 3:00 A.M. over the midyear holiday break from school. To ensure validity of the prepared vignette, students were asked to report if the described situation was realistic for them. If not, they were asked to report if they could easily imagine themselves participating in the situation. If neither of these conditions existed for a particular individual, that person was eliminated from the potential participant pool.

\section{Participants}

The participants for this study were chosen from the population of traditional-aged ( 17 to 24 years) female undergraduate students at a state university in the southeastern United States. A power analysis was conducted, and indicated that with a total sample size of $40(20$ COAs and 20 CONAs) and statistical power of 80 would be obtained. This level of statistical power was assessed as appropriate, and the final sample included 27 COAs and 76 CONAs enrolled in two undergraduate counseling classes.

\section{Instruments}

Three instruments were used in this study: The Children of Alcoholics Screening Test (CAST) (Jones, 1983a), the BDI, and the WCCL-R. In addition, demographics information was collected to assess age and sex, serving as sample selection factors.

Children of Alcoholics Screening Test (CAST). The CAST, a 30item inventory measuring attitudes, feelings, perceptions, and experiences related to parental drinking was used in conjunction with self-report to screen for the presence of parental alcoholism and to assign participants to the COA and CONA groups. The CAST was designed to yield one score, ranging from 0 to 30 . Participants were assigned to groups on the basis of recommended cut-scores listed within the CAST manual (Jones, 1983a). A CAST score of greater than 5 was suggested for the purposes of identifying COAs. In addition, individuals were required to self-report the presence of parental alcoholism to be placed in the COA group. Individuals with inconsistent ratings on the two measures of parental alcoholism (the CAST and self-report) were eliminated from participation in the study.

The CAST was normed using individuals from age 9 through adulthood (Jones, 1983a, 1983b). Split-half reliability for the CAST was .98 for a sample of 133 latency-age and adolescent individuals. Validity was established on 82 children of clinically-diagnosed alcoholics, 15 self-reported COAs, and 118 randomly selected CONAs (Jones, 1983b). Using the method of contrasted groups, an analysis of variance revealed that the children of clinically diagnosed alco- holics and self-reported COAs scored significantly higher when compared with the control group $(F(2,21)=166.5, p<.0001)$. Additionally, chi-square analyses revealed that all 30 CAST items effectively discriminated COAs from CONAs.

The Beck Depression Inventory $(B D I)$. Each participant's level of depression was measured with the $\mathrm{BDI}$, the most frequently used measure of depression in the literature on both COAs and coping. Internal consistency ratings for the BDI (Cronbach's alpha) ranges from .79 to .90 for varying samples (Beck \& Steer, 1984). Validity coefficients range from .38 to .76 when the BDI is compared with the construct of hopelessness and a variety of alternative measures of depression.

The Ways of Coping Checklist-Revised (WCCL-R). The WCCL$R$, a 66-item questionnaire assessing reactions to specific stressful encounters, is a measure of coping derived from Lazarus's transactional theory of stress and coping. A factor analysis from a study of college students coping with a midterm examination yielded eight distinct coping strategies (Folkman \& Lazarus, 1985): problemfocused coping, wishful thinking, detachment, seeking social support, emphasizing the positive, blame-self, tension reduction, and selfisolation. The WISC-R was administered twice, once in response to the actual stressful encounter, and once using the prepared vignette.

Coefficient alphas for the WCCL-R range from .73 to .88 using samples of medical students, spouses of patients with senile dementia, and psychiatric outpatients (Vitaliano, Russo, Carr, Maiuro, \& Becker, 1985). Construct validity was established through associations between the subscales of the WCCL-R and several measures of anxiety and depression.

\section{RESULTS}

\section{Differences in Level of Depression}

An independent two-sample $t$ test applied to the BDI scores indicated that the mean COA group score $(M=7.96, S D=7.66)$ was significantly higher than that obtained for the CONA group $(M=4.49$, $S D=5.17$ ). Nevertheless, mean scores for both groups were within the no depression range as described in the BDI test manual. A total of 21 of the COA participants $(78 \%)$ and 66 of the CONAs $(87 \%)$ scored within the no depression range. An additional 4 COAs (15\%) and 8 CONAs $(11 \%)$ scored within the mild-moderate range. Only 1 of the COA participants (4\%) and 2 CONAs (3\%) scored within the moderate-severe range, and $1 \mathrm{COA}(4 \%)$ scored within the severe depression range on the $\mathrm{BDI}$.

\section{Differences in Coping Strategy Profiles}

Pearson product-moment correlations were calculated between WCCL-R subscale scores obtained in response to the actual stressful encounter and those obtained in response to the prepared vignette $(r=.25$ to .59$)$. Due to the relatively weak relationship between subscale scores on the two administrations, it was decided that results would be analyzed separately for these scores.

A multivariate analysis of covariance (MANCOVA) was applied to subscale scores of the WCCL-R, controlling for level of depression. In response to the actual stressful encounter, an overall difference was detected between COAs and CONAs on their use of the eight coping strategies under study, $F(8,91)=2.13, p<.05$. The use of Bonferonni's correction to adjust for the application of eight follow-up univariate tests limited the significance level to .0063. Under these conditions, no differences were detected between the two groups on the use of individual coping strategies. 
In response to the prepared vignette, no overall differences were detected between the COA group and the CONA group on their use of the eight coping strategies under study, $F(8,92)=1.01, p<.44$.

\section{Discriminatory Analysis}

Results of the discriminatory analysis indicated that the use of depression and the eight coping strategies under study for discriminating between COAs and CONAs is prohibitive. The derived models were fairly accurate in predicting CONA group placement; however, the percentage of misclassifications was high for the COA group.

In response to the actual stressful encounter reported by participants, depression and self-isolation were included in the final discriminatory model, $F(2,99)=5.79, p<.01$, and the associated discriminant function was: $-2.94+.07(\mathrm{BDI})+.69$ (self-isolation). In response to the prepared vignette, after depression was forced into the model, no other variables met the criteria for inclusion. Therefore, group discrimination in response to the prepared vignette was based on depression only, and the discriminant function was: $-1.63+$ $10(\mathrm{BDI})$

It was expected, based on prior probabilities, that $26 \%$ of the sample would be predicted to be COAs and that $74 \%$ would be predicted to be CONAs. Results of the discriminant analysis on WCCL$\mathrm{R}$ responses to the actual stressful encounter, however, indicated that 5 of the 27 COAs under study (19\%) were correctly predicted to be COAs, but $22(81 \%)$ were incorrectly predicted to be CONAs. Of the other participants, 72 (95\%) CONAs were correctly predicted to be CONAs, whereas $4(5 \%)$ were incorrectly predicted to be COAs.

In response to the prepared vignette, the discriminant analysis of the WCCL-R scores showed 2 of the COAs (7\%) to be correctly classified on the basis of the derived model, but 25 (93\%) were incorrectly classified as CONAs. For the CONAs, $4(5 \%)$ were incorrectly classified as COAs, whereas $72(95 \%)$ were correctly classified.

\section{DISCUSSION}

The findings of this study must be discussed with some limitations in mind. Due to logistical considerations, the sample for this study was not randomly selected and consisted of volunteers. Therefore, it may not be representative of the general population of either COAs or CONAs. In addition, interpretations of the findings of the WCCL$\mathrm{R}$ must be made with caution. On the administration of the WCCL$\mathrm{R}$ given in response to the actual stressful encounter reported by participants, it is important to note that each participant was responding to a unique stressful encounter. Although all of the events took place within the context of the family, differences noted may relate to differences in the nature of the stressful encounters described. In addition, the dependent variables (the BDI and the WCCL-R) may be susceptible to response bias for the population under study, and might lack the sensitivity required to detect the kinds of differences hypothesized to be present between COAs and CONAs on the characteristics under study.

The results of this study lead to several possible explanations and implications. For example, the significant difference detected between COAs and CONAs in level of depression is consistent with existing research (Calder \& Kostiniuk, 1989; Tweed \& Ryff, 1991). However, the fact that statistically significant differences were noted does not accurately reflect the nature of the actual distribution of BDI scores for this particular sample. Mean BDI scores for both groups was within the range of "no depression," and the overall distribution of BDI scores for both groups was similar. The more notable difference was in the variability of BDI scores for both groups; the scores for the COA group were more variable than those for the CONA group. These findings suggest that unequivocal claims that COAs are more depressed than CONAs might be misleading. The use of specific score distributions as an overall framework may assist in a more accurate interpretation of detected group differences.

A review of the actual stressful encounters provided by participants revealed discrepancies not detected within statistical analyses. COA participants were far more likely than CONAs to report incidents that a counselor might associate with elevated levels of depression. For example, one COA reported that the most stressful encounter that took place within her family within the past 6 months was the suicide of her mother. She indicated that this event had taken place 3 weeks before the data collection process, and that she had discovered the body. Another COA reported that she had witnessed her father "beat her sister while in an alcoholic rage." Still another COA had been hit by her alcoholic father on the night prior to data collection. Each of these individuals scored 0 (no depression) on the BDI. Although a vast majority of BDI scores for the CONA group fell within that range, incidents with this level of associated tragedy were not typical among the group. More typical were descriptions of stressful encounters related to financial insecurity in the family, unsatisfactory grades in school, or parental dissatisfaction over life-style choices (e.g., boyfriends, living arrangements) made by participants.

Although no significance can be attributed to group differences in the quality of stressful encounters provided, this may represent an area for future study. Several hypotheses exist that might explain why COAs do not seem to experience depression in the face of events that seem to be quite traumatic. The more popular and clinically based literature on COAs suggests that these individuals may use a great deal of denial in coping with trauma (Beattie, 1987; Black, 1981). It is also speculated within this literature that COAs may be accustomed to discomfort and chaos, and may dissociate in response to high levels of trauma and distress.

Differences between COAs and CONAs in their use of the eight coping strategies under study were not found to be statistically significant. As a result, the derived prediction models included only one coping strategy. In response to the actual stressful encounter, depression and self-isolation were chosen to predict group placement, and for the prepared vignette, only depression was used to discriminate COAs from CONAs. Although the derived models did a good job of accurately predicting group placement for the CONA group, none of the variables under study accurately predicted group placement for the COA group. This finding occurred as a result of two separate occurrences within the data. The nonsignificant differences in choice of coping strategies between COAs and CONAs accounted for the absence of specific coping strategies in the derived models. In addition, the actual lack of depression present within the COA group explains the high percentage of misclassifications for this group. Although statistical analyses supported elevated level of depression among the COA group, the majority (78\%) of these individuals were not depressed according to BDI scores. These findings support the use of caution in applying conclusions based on statistical findings alone.

The results of this study most apparently support the need for more studies of depression among children of alcoholics. Depression scores among the COA group were shown to be quite variable, and the derived discriminatory models were not sufficient to accurately predict which participants were COAs. Also, the vast differences between COAs and CONAs in the quality of stressful encounters described further support the need for more COA group studies. Although the kinds of events reported by COAs were overwhelm- 
ingly more tragic in nature, the majority of COA participants were not depressed. In addition, they did not cope differently than did their CONA cohorts. One possible explanation for the lack of differences in choice of coping strategies may relate to developmental issues associated with the chosen sample. Developmental theory (Bandura, 1964; Blos, 1941; Erikson, 1950) suggests that the primary task associated with individuals within the 17- to 24-year age range revolves around the formation of an identity. It is speculated that a part of this process involves the emergence of the peer group as a primary source of direction and identification. As a result, many adolescents strive to be perceived as "like" their peer group, and this powerful developmental pull may preclude honesty in the assessment of individual characteristics or differences. In addition, the homogeneity within the sample, including the fact that only college students were assessed, may assist in explaining the lack of detected group differences.

For the practitioner, the implications of this study confirm what is shown throughout the literature on COAs. To date, no empirically based, firm characterization of this population is available. In addition, the application of depression-specific treatment strategies, based on the overwhelming evidence throughout the literature suggestive of higher levels of depression among COAs, is not supported in this study.

In this study, the discrepancy between the quality of actual stressful encounters reported by COA participants and associated levels of depression might lead counselors to carefully consider the issue of assessment of COA clients. It seems that the more traditional methods of self-report might not supply information sufficient to diagnosis psychological symptomatology for this population. The results of the self-report depression measure did not suggest that depression is a predominant issue for this population. This evidence alone suggests that counselors use caution in drawing conclusions related to level of depression based solely on the presence of parental alcoholism. Nonetheless, the quality of actual stressful encounters reported by the COA clients suggests otherwise. Certainly, a client scoring 0 on any measure of depression who simultaneously indicates that they have experienced the suicidal death of a parent within the past several months warrants an in-depth assessment of potential psychological symptoms.

Clearly, the treatment of COA clients remains complex and seemingly individual in nature. The differences shown to exist within this group most clearly support the need for flexibility and variety in working with this population.

\section{REFERENCES}

Bandura, A. (1964). The stormy decade: Fact or fiction? Psychology in the Schools, 1, 224-231.

Beattie, M. (1987). Codependent no more. New York: Harper \& Row.

Beck, A. T., \& Steer, R. A. (1984). Internal consistencies of the original and revised Beck Depression Inventory. Journal of Clinical Psychology, 40, 1365-1367.

Beck, A, T., Ward, C. H., Mendelson, M., Mock, J., \& Erbaugh, J. (1961). An inventory for measuring depression. Archives of General Psychiatry, 4, $561-571$.
Berkowitz, A., \& Perkins, H. W. (1988). Personality characteristics of children of alcoholics. Journal of Consulting and Clinical Psychology; 56, 206-209. Black, C. (1981). It will never happen to me. New York: Ballantine.

Blos, P. (1941). The adolescent personality: A study of individual behavior. New York: Appleton-Century.

Borgatta, E. F. (1965). A short test of personality: The S-ident Form. Journal of Educational Research, 58, 453-456

Calder, P., \& Kostiniuk, A. (1989). Personality profiles of children of alcoholics. Professional Psychology: Research and Practice, 20, 417-418.

Erikson, E. H. (1950). Childhood and society. New York: Norton

Folkman, S., \& Lazarus, R. S. (1985). If it changes it must be a process: A study of emotion and coping during three phases of a college examination. Journal of Personality and Social Psychology, 48, 150-170.

Jones, J. W. (1983a). The children of alcoholics screening test: Test manual. Chicago: Camelot Unlimited.

Jones, J. W. (1983b). Psychometrically identifying grown-up children of alcoholics. Unpublished manuscript.

Kolenc, K. M., Hartley, D. L., \& Murdock, N. L. (1990). The relationship of mild depression to stress and coping. Journal of Mental Health Counseling. 12, 76-92.

Lazarus, R. S., \& Folkman, S. (1984). Stress, appraisal. and coping. New York: Springer.

Lazarus, R. S., \& Launier, R. (1978). Stress-related transactions between person and environment. In L. A. Pervin \& M. Lewis (Eds.), Perspectives in interactional psychology. New York: Academic.

Martin, A. (1991, April). Perceived self-efficacy and coping behaviors related to the stress of family relationships among adult COAs. Paper presented at the International Family Nursing Conference, Portland, OR.

National Institute on Alcohol Abuse and Alcoholism. (1985, October). Children of alcoholics. Alcohol Resources: Update. Rockville, MD.

Pearlin, L. I., \& Schooler, C. (1978). The structure of coping. Journal of Health and Social Behavior, 19, 2-21.

Plescia-Pikus, M., Long-Sutter, E., \& Wilson, J. P. (1988). Achievement, wellbeing, intelligence, and stress reaction in adult children of alcoholics. Psychological Reports, 62, 603-609.

Prewett, M. J., Spence, R., \& Chaknis, M. (1981). Attribution of causality by children with alcoholic parents. International Journal of the Addictions, 16 . 367-370.

Russell, M., Henderson, C., \& Blume, S. B. (1985). Children of alcoholics: A review of the literature. New York: Children of Alcoholics Foundation.

Scavnicky-Mylant, M. (1990). The process of coping among adult children of alcoholics. Issues in Mental Health Nursing, II, 125-139.

Svanum, S., \& McAdoo, W. G. (1991). Parental alcoholism: Examination of male and female alcoholics in treatment. Journal of Studies on Alcohol, 52 , $127-132$.

Thompson, S. C. (1981). Will it hurt if I control it? Complex answer to a simple question. Psychological Bulletin, 90, 89-101.

Tweed, S. H., \& Ryff, C. D. (1991). Adult children of alcoholics: Profiles of wellness amidst distress. Journal of Studies on Alcohol, 52, 133-141.

Vitaliano, P. P., Russo, J., Carr, J. E., Maiuro, R. D., \& Becker, J. (1985). The ways of coping checklist: Revision and psychometric properties. Multivariate Behavioral Research, 20, 3-26.

Virginia A. Kelly is an assistant professor in the Division of Human Services at the University of Cincinnati. Jane E. Myers is a professor in the Department of Counseling and Educational Development at the University of North Carolina at Greensboro. Correspondence regarding this article should be sent to Virginia A. Kelly, The State University of New York at Plattsburgh. Center for Human Services, 107 A Ward Hall, Plattsburgh, NY 12901. 